\title{
Femoral Head Avascular necrosis following intramedullary Gamma-nailing: A rare complication.
}

\author{
Themistoklis Vampertzis ${ }^{1}$, Ioannis K. Christou ${ }^{1}$, Paraskevas Giannakopoulos ${ }^{1}$, Christina Barmpagianni², \\ Georgios Nitis' ${ }^{1}$, Athanasios Fotiadis ${ }^{1}$, Stergios Papastergiou ${ }^{1}$
}

'Department of Orthopaedics - Unit for Sport Injuries, Agios Pavlos General Hospital of Thessaloniki, Thessaloniki, Greece; ${ }^{2}$ Trauma and Orthopaedics, Northwick Park Hospital, London North West University Healthcare NHS Trust, United Kingdom

\begin{abstract}
Avascular necrosis of the femoral head affects many patients annually. It results from reduced blood supply and can lead to complete loss of joint. The development of such a complication after certain types of hip trauma such as neck of femur fractures is not uncommon; nevertheless, extra-capsular fractures such as intertrochanteric ones are not a frequent cause of this complication reported at $0.5 \%$. This case report presents the case of a 76 year-old woman who developed femoral head avascular necrosis following the fixation of her intertrochanteric fracture by an intramedullary Gamma nail. The early post-operative period suggestive of full recovery was followed by newlydeveloped symptoms of pain and reduced mobility. The removal of implants only partially alleviated symptoms while avascular necrosis was noted afterwards and was treated by a Total Hip Replacement before progression to involve the acetabulum. What is significant to stress in this case is the rarity of this complication arising after the specific trauma and operative technique. The anatomical location of the fracture does not advocate for a disruption in the femoral head's perfusion, neither does the fixation by an intramedullary approach. Nevertheless physicians should always be aware of such cases and investigate all patients with post-operative pain and/or reduced range of motion for avascular necrosis, as early recognition is essential.
\end{abstract}

Keywords: Avascular Necrosis of femoral head, Intertrochanteric fracture, Intramedullary gamma nail, Total hip replacement, Femoral head blood supply

\section{Introduction}

Avascular Necrosis of the femoral head is a condition of variable etiology that affects up to 20,000 patients of all age groups annually ${ }^{1}$. It is a pathological state associated with the reduction of vascular supply to the subchondral bone of the femoral head leading to osteocyte death and the inevitable collapse of the affected femoral head ${ }^{2}$. Predisposing factors include but are not limited to fracture, steroids, alcohol abuse, metabolic disorders and haemoglobinopathies ${ }^{2-5}$. Initially, the disease is clinically silent therefore at presentation destruction of the bone is often substantial ${ }^{2}$. Treatment includes non-invasive management with pain control and reduced weight-bearing but a surgical intervention is in most cases inevitable ${ }^{1,6}$.

Of the traumatic etiologies of Avascular Necrosis (AVN) of the femoral head intertrochanteric fractures are an infrequent cause of such complications $(0.5 \%)^{7}$. The reason is that neither the fracture itself nor the fixation method are affecting the blood supply to the head of femur ${ }^{7,8}$. The femoral head is nourished by three arteries: the nutrient artery of the shaft, the retinacular/capsular arteries and the artery of the round ligament of femur/foveolar artery ${ }^{8}$.

This case report presents the development of AVN of the femoral head after intramedullary Gamma nailing of an intertrochanteric fracture in an elderly female patient.

The authors have no conflict of interest.

Corresponding author: Themistoklis Vampertzis, MD, Department of Orthopaedics - Unit for Sport Medicine, Agios Pavlos G.H., Ethnikis Antistaseos 161, 55134 , Thessaloniki, Greece

E-mail: themisvamper@yahoo.com

Edited by: Christos Yiannakopoulos

Accepted 8 May 2020 


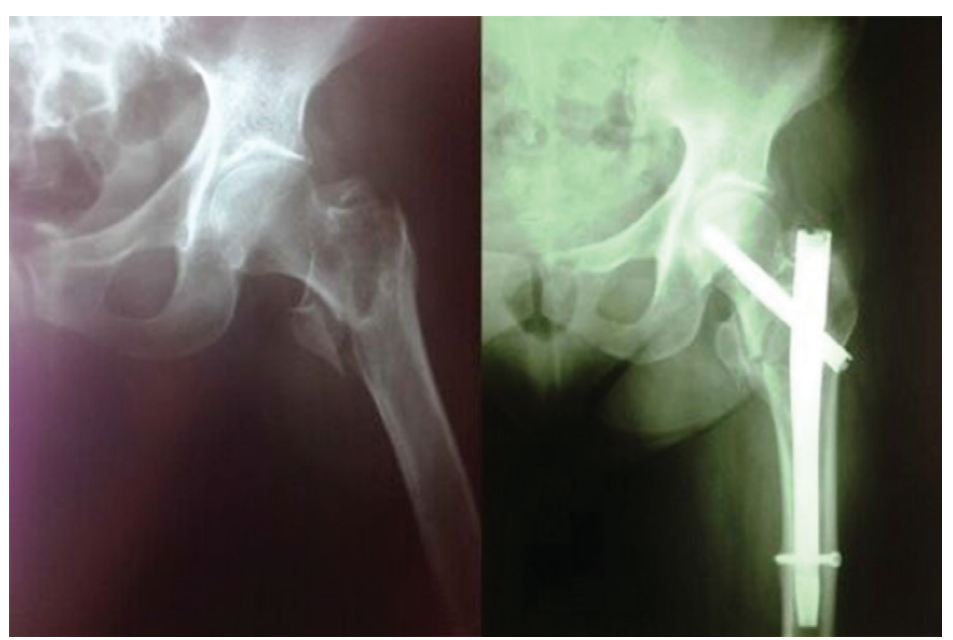

Figure 1. Intertrochanteric fracture of the left hip (left) treated with intramedullary gamma nail on the post-operative X-ray (right).

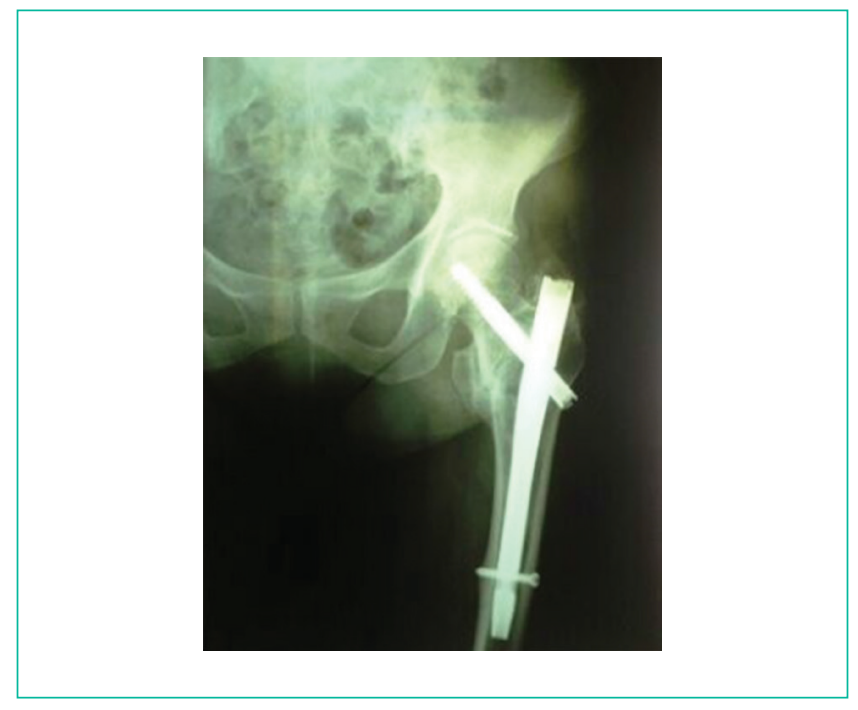

Figure 2. 6 months post-operative after intramedullary gamma nail.

\section{Case description}

A 76 year-old female sustained an intertrochanteric fracture of her left hip after a low energy trauma. She was treated with intramedullary Gamma nailing, while the postoperative radiographic control showed that the reduction was successful and the implant position acceptable (Figure 1). The patient recovered without any post-operative complications and was compliant with the protocol for partial weight-bearing on the left leg using a walking frame. On the third post-operative month she could walk unassisted. Radiographic control six months following the operation showed union of the fracture (Figure 2).

Eight months after the operation the patient visited the Outpatient Department with complains of pain while walking managed by over the counter analgesic medication. One year after the operation the implants were removed due to increasing pain and reduced Range of Motion on the left hip. This intervention helped with pain control while mobility was not affected significantly. The patient was followed-up regularly and 6 months later early stage Avascular Necrosis of the left femoral head was identified on X-rays. At this stage conservative management was advised with partial weight-bearing and use of walking aid while planning for a new surgery. Due to the progression of the AVN over the next 6 months (increasing pain and inability to mobilize unassisted) the decision was to treat her operatively. Because of the irreversibility of AVN the best solution was a Total Hip Arthroplasty after which she recovered well and was able to once again mobilize unassisted (Figure 4).

\section{Discussion}

Avascular necrosis of the femoral head is a progressive clinical condition of multifactorial etiology and variable presentation. It affects all age groups including the pediatric population and its progression to necrosis and femoral head collapse is often irreversible ${ }^{2}$. AVN is a consequence of prolonged ischemia i.e. it arises when blood supply to the bone is interrupted ${ }^{2}$. This can be either due to a macroscopic damage to vessels as in the case of trauma/fracture or due to microscopic vascular changes that can be attributed to autoimmune disorders, metabolic diseases, use of steroids while it can also be idiopathic in origin ${ }^{2-5}$. By the time clinical symptoms are noticed by patients the damage to the bone 


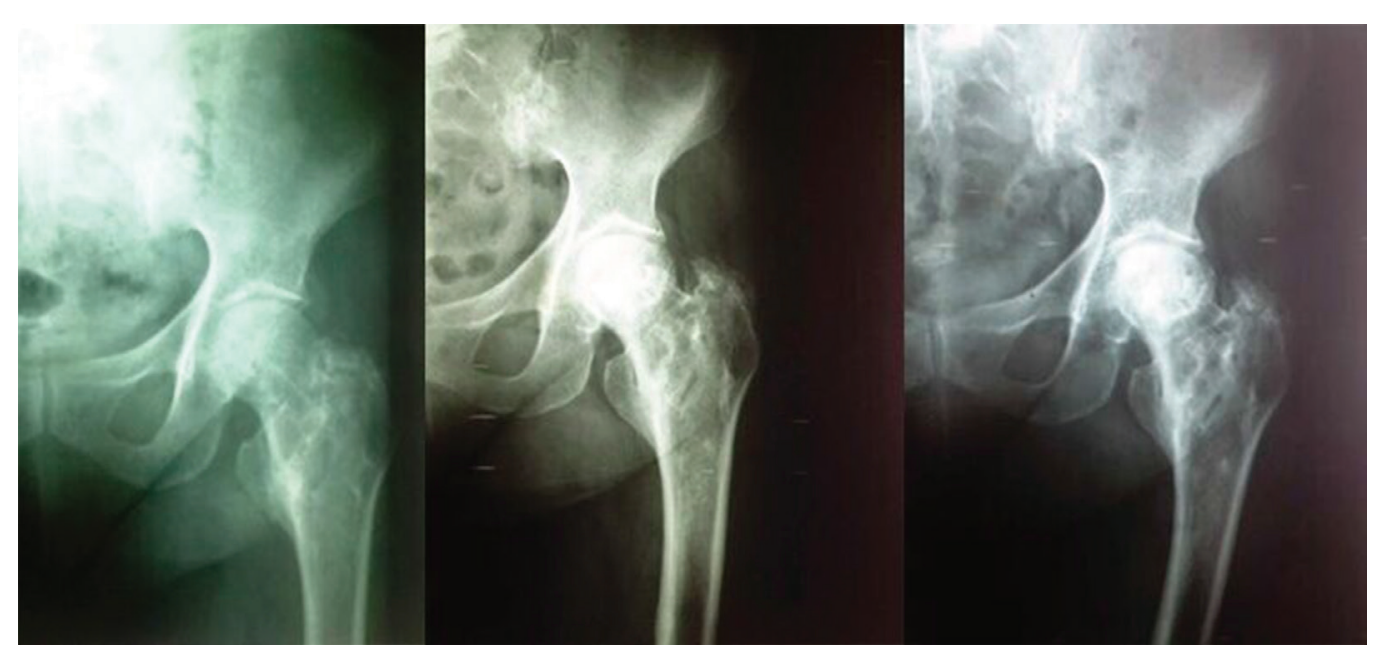

Figure 3. Removal of implants during the $12^{\text {th }}$ post-operative month (left); development of left femoral head AVN 6 months after removal of implants (middle); left femoral head AVN 12 months after removal of implants (right).

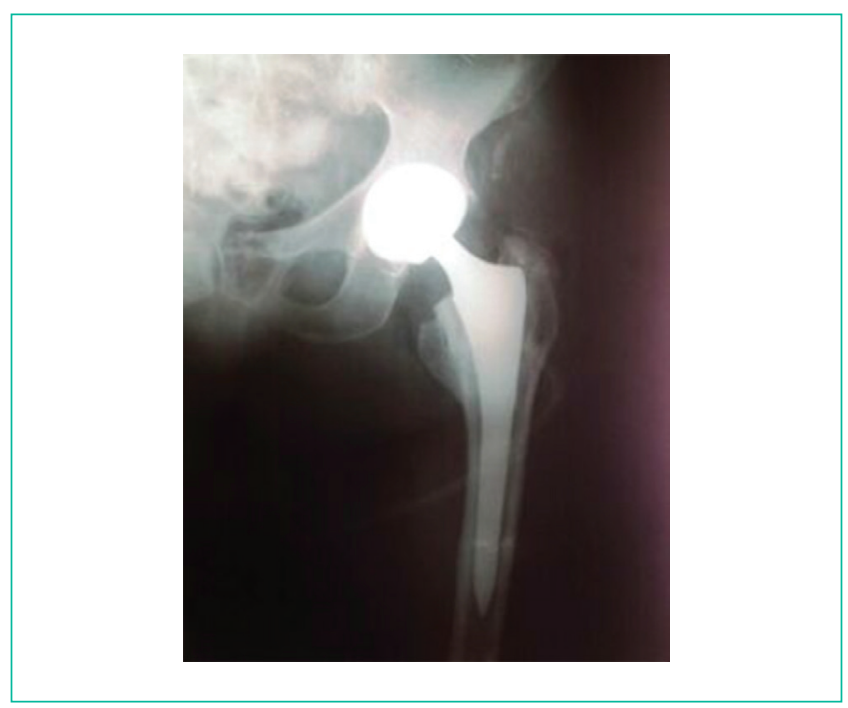

Figure 4. Left Total Hip Arthroplasty 5 years post operatively.

is significant ${ }^{2}$. Patients frequently complain of pain with or without decrease in Range of Motion and reduced mobility ${ }^{2}$. Once suspected AVN can be diagnosed by plain X-rays or Magnetic Resonance Imaging ${ }^{2}$. The choice between conservative versus surgical treatment is made relatively to the stage of disease at presentation and the rate of progression in follow-up examinations ${ }^{6}$.

This report discusses the case of a 76 year-old woman who developed AVN of the femoral head after an intertrochanteric fracture treated by intramedullary Gamma nailing. This is a quite rare complication reported at a $0.5 \%$ occurrence due to the fact that neither the mechanism/location of the fracture nor its fixation are known to affect nourishment of the femoral head from its three main supplying arteries (nutrient artery of the shaft; retinacular/capsular arteries; artery of the round ligament of femur/foveolar) ${ }^{7,8}$. A Total Hip Replacement which is the most advisable treatment in such cases as per many authors was performed ${ }^{5-7}$. The result was successful and the patient regained the full function of her limb.

Concluding, avascular necrosis of the femoral head is a rare complication of intertrochanteric fractures but still an important one to be aware of. High risk patients should have a longer follow-up in order to identify and treat AVN in early stages before the development of hip osteoarthritis and the permanent erosion of the acetabulum.

\section{References}

1. Shannon BD, Trousdale RT. Femoral osteotomies for avascular necrosis of the femoral head. Clin Orthop Relat Res 2004;418;34-40.

2. Guerado E, Caso E. The physiopathology of avascular necrosis of the femoral head: an update. Injury 2016;47(6);S16-S26.

3. Ehlinger M, Moser T, Adama P, Bierry G, Gangi A, de Mathelin M et al. Early prediction of femoral head avascular necrosis following neck fracture. Orthop Traumatol Surg Res 201 1;97(11);79-88.

4. Vicario C, Marco F, Ortega L, Alcobendas M, Dominguez I, LodezDuran $L$. Necrosis of the femoral head after fixation of trochanteric fractures with Gamma Locking Nail: A cause of late mechanical failure. Injury 2003;34(2); 129-134.

5. Deleanu B, Prejbeanu R, Vermesan D, Honcea L, Mioc Lazar M, Tsiridis $\mathrm{E}$ et al. Avascular necrosis of the femoral head at 2 years 
after peritrochanteric fracture surgery: Case report. Ann Med Surg 2016;5;106-109.

6. Mohanty SP, Singh KA, Kundangar R, Shankar V. Management of non-traumatic avascular necrosis of the femoral head - a comparative analysis of the outcome of multiple small diameter drilling and core decompression with fibular grafting. Musculoskelet Surg
2017; 101(59);59-66.

7. Bojan AJ, Beimel C, Speitling A, Taglang G, Ekholm C, Jonsson A. 3066 consecutive Gamma Nails. 12 years experience at a single centre. BMC Musculoskelet Disord 2010;11;133.

8. Tucker FR. Arterial supply at the femoral head and its clinical importance. J Bone Joint Surg Br 1949;31B(1);82-93. 